\title{
Subjective versus objective measurement of surgical outcomes of treatment of female stress urinary incontinence: it is not just black and white
}

\author{
Berna Haliloglu • Diaa E. E. Rizk
}

Received: 19 March 2010/Accepted: 26 March 2010/Published online: 4 May 2010

(C) The International Urogynecological Association 2010

The best interest of the patient is the only interest to be considered.

William J. Mayo

Stress urinary incontinence (SUI) is encountered in as many as $20 \%$ of women with adverse social and hygienic consequences [1]. Recently, most studies have shown that the majority of surgical procedures, particularly the minimally invasive midurethral slings, are both safe and effective treatment of female SUI. However, the success rates reported varied between studies, depending on whether objective or subjective outcomes had been measured. The postoperative objective and subjective cure rates were not also correlated in most of these studies. For example, a recent review gives a very wide range of short-term success rates of various surgical procedures for treatment of female SUI, from $32 \%$ to $100 \%$ [1]. It is extremely important that comparison of the results of surgical treatment of SUI between different surgeons and centers as well as patient counseling is based on robust scientific evidence that includes valid and accurate assessment of the treatment outcome. It seems timely, therefore, to discuss this topic further mainly by addressing

\section{B. Haliloglu}

Department of Obstetrics and Gynecology, Faculty of Medicine, Maltepe University,

Istanbul, Turkey

\section{E. E. Rizk}

Department of Obstetrics and Gynecology, Faculty of Medicine, Ain Shams University,

Cairo, Egypt

\section{B. Haliloglu ( $\square)$}

Ataturk Cd. Cam Sk. 3/A 34843, Maltepe,

Istanbul, Turkey

e-mail: bernadr23@hotmail.com

e-mail: bernahaliloglu@yahoo.com three main questions: What do we mean by surgical cure of SUI? How do we measure this cure? What is the definition of success of operative intervention for SUI?

There has been a lack of consensus in the urogynecologic community about which postoperative outcome measure to use to evaluate the efficacy of surgical procedures for SUI. Castillo et al. [2] analyzed 91 surgical series of SUI and reported that $33 \%$ of these studies used only subjective measures, $4 \%$ only objective measures, and $63 \%$ both objective and subjective measures, for the definition of "cure." Not surprisingly, therefore, the cure rates of the same surgical procedure for SUI differed among these studies. A universal definition of cure is thus obviously needed for accurate interpretation of cure rates when surgical treatment of SUI is contemplated.

Surgeons tend to define the cure of SUI as the successful anatomical repair of defective tissue support of the urethra measured by objective improvement in urinary incontinence. As a direct result, earlier studies of surgical treatment of SUI focused on objective outcomes for measurement and definition of "surgical" cure. However, these measures tend to underestimate the impact of lower urinary tract symptoms on social limitations and emotional well-being of the woman [3]. Patients may have completely different expectations of cure after surgery for SUI other than the anatomical cure, such as absence of odor, lack of disruption of routine activities, use of less pads, and/or improvement of emotional status. For patients, the improvement of these symptoms might be more important than the "objective" demonstration of leakage of urine. In fact, a number of patients who are considered as objectively cured from SUI might even feel worse following the operation due to surgery-induced negative outcomes such as de novo urge urinary incontinence or intermittent selfcatheterization because of voiding dysfunction. Conversely, some patients who are considered not to be objectively cured by surgeons are satisfied with the outcome following the 
operation. Furthermore, the measurement validity of some objective outcomes such as voiding diaries and pad test may be limited by patient's compliance. This difference in perceptions of success of operative intervention between surgeons and patients may explain the discordant cure rates for surgical treatment of SUI reported in the literature [4].

SUI is a not a life-threatening disorder but has a significant adverse impact on quality of life, and therefore treatment should be dictated by the preferences of women. This is usually influenced by many extrinsic non-treatment-related factors such as the previous experience of family members and friends with treatment of SUI or the patient's own past experience with other medical disorders necessitating surgical intervention. Thus, the patient's perspective concerning surgical intervention is the key point to be addressed when considering surgical treatment of SUI. Brubaker and Shull [5] described the new concept of patient-centered outcomes using the EGGS acronym (E: expectations, G: goal setting, G: goal achievement, S: satisfaction) that should to be routinely assessed before any operations for SUI. They recommended that the overall satisfaction of patients should be the most important measure to assess the postoperative outcome of anti-incontinence surgery since objective outcome measures may not reveal the full impact of a surgical procedure on a patient's physical well-being, social function, mental health, societal role, and sexual health. The overall patient satisfaction should be reassessed at frequent intervals in the postoperative period since this is known to deteriorate with time. For example, a patient may feel satisfied in the first few weeks after anti-incontinence surgery because she has significantly less incontinence episodes. However, in the longterm, she may become bothered by pain during intercourse as a result of the operation, resulting in less satisfaction.

Most women with SUI suffer from symptoms that often leave them uncomfortable, embarrassed, and socially withdrawn but are often reluctant to discuss these concerns with their physicians. Self-esteem and body image may be altered by underwear wetting, skin irritation, and urinary odor or by the need for frequent use of pads. In some cases, patients are also forced to adopt alternative strategies such as restriction of fluid intake, toilet mapping, and avoidance of sexual intimacy. Indeed, urinary incontinence, irrespective of its cause, is often perceived by women as a stigma of loss of self-control. Therefore, determination of success of surgical procedures for SUI according to "subjective" expectations of patients based on quality-of-life assessment has gained increasing attention in recent studies. When these expectations are not met, the postoperative outcome and patient satisfaction might be adversely affected. However, quality-of-life questionnaires may still fail to detect the primary postoperative outcome of surgery - cure/improvement of incontinence - and may not be related to the patient's overall clinical condition [4]. Although the addition of global impression questionnaires for incontinence will allow detection of individual response to surgery, the use of these indices with other quality-of-life questionnaires as a postoperative outcome measure of surgical treatment of SUI is not yet established [6].

It is axiomatic that any surgical procedure for SUI should identify and aim to repair the specific anatomical defects in urethral support. This is the "objective" success rate that reflects the success of the procedure and the surgeon. However, women's expectations and satisfaction also have a substantial importance in determining the "subjective" success rate that reflects the patient's perception of success of surgical intervention. For this reason, neither objective nor subjective cure rates should be used alone. So far, there has been no single measure that reflects the objective or subjective postoperative outcome of any surgical procedure, and those used for treating SUI are a case in point.

In conclusion, studies attempting to bridge the gap between patient perception of surgical treatment and objective postoperative outcomes of SUI are awaited with interest. Until these data are available, there is an urgent need for a novel "gold standard" outcome measure that includes both the patient's expectations and surgeon's assessment and assigns them equal importance. While the objective measures demonstrate the ability to correct anatomical defects at operation, representing the likelihood of success of the surgeon and procedure, subjective improvement in postoperative quality of life reflects the satisfaction of the individual patient with the surgical procedure. To paraphrase our debate, as a urogynecologist provider, which measure would you prefer to evaluate the cure and/or success of surgical treatment of SUI: your choice of the operative technique and surgical skills, the woman's satisfaction and perception of improvement, or both?

\section{Conflicts of interest None.}

\section{References}

1. Padmanabhan P, Nitti VW (2006) Female stress urinary incontinence: how the patient and physician perspective correlate in assessment of outcomes? Curr Opin Urol 16:212-218

2. Castillo PA, Espaillat-Rijo LM, Davila GW (2010) Outcome measures and definition of cure female stress urinary incontinence surgery: a survey of recent publications. Int Urogynecol J 21:343-348

3. Srikrishna S, Robinson D, Cardozo L, Gonzalez J (2009) Is there a discrepancy between patient and physician quality of life assessment? Neurourol Urodyn 28:179-182

4. Ghoniem G, Stanford E, Kenton K, Achtari C, Goldberg R, Mascarenhas $\mathrm{T}$ et al (2008) Evaluation and outcome measures in the treatment of female urinary stress incontinence: International Urogynecological Association (IUGA) guidelines for research and clinical practice. Int Urogynecol J 19:5-33

5. Brubaker L, Shull B (2005) EGGS for patient-centered outcomes. Int Urogynecol J 16:171-173

6. Yalcin I, Bump RC (2003) Validation of two global impression questionnaires for incontinence. Am J Obstet Gynecol 189:98-101 